Case Report

\title{
Therapeutic Effect of Anti-VEGF for Age-Related Macular Degeneration in the Untreated Fellow Eye
}

\author{
Hacer Isildak $(\mathbb{D}),{ }^{1}$ Stephen G. Schwartz ${ }^{(D)},{ }^{1}$ and Harry W. Flynn Jr. ${ }^{2}$ \\ ${ }^{1}$ Department of Ophthalmology, Bascom Palmer Eye Institute, University of Miami Miller School of Medicine, \\ 3880 Tamiami Trail N., Naples, FL 34103, USA \\ ${ }^{2}$ Department of Ophthalmology, Bascom Palmer Eye Institute, University of Miami Miller School of Medicine, \\ 900 NW 17th St., Miami, FL 33136, USA \\ Correspondence should be addressed to Stephen G. Schwartz; sschwartz2@med.miami.edu
}

Received 9 February 2018; Accepted 18 March 2018; Published 24 April 2018

Academic Editor: J. Fernando Arevalo

Copyright ( $\odot 2018$ Hacer Isildak et al. This is an open access article distributed under the Creative Commons Attribution License, which permits unrestricted use, distribution, and reproduction in any medium, provided the original work is properly cited.

Intravitreal injections of antivascular endothelial growth factor (anti-VEGF) agents have been reported to occasionally produce a therapeutic effect in the uninjected fellow eye. Here, three patients with bilateral neovascular age-related macular degeneration are presented. In all three patients, unilateral anti-VEGF injection resulted in bilateral reduction of macular thickness as measured by spectral domain optical coherence tomography.

\section{Introduction}

The use of antivascular endothelial growth factor (antiVEGF) agents is standard treatment for neovascular agerelated macular degeneration (AMD), diabetic macular edema (DME), and other disorders [1,2]. Several studies have reported bilateral therapeutic effects following unilateral anti-VEGF injection in patients with AMD [3-5], DME [6$11]$, and other disorders.

Here, three patients with bilateral neovascular AMD are presented in which unilateral injection of anti-VEGF agents resulted in bilateral reduction of macular thickening as measured by spectral domain optical coherence tomography (SD-OCT). Institutional Review Board approval was not required for this study.

\section{Case Reports}

2.1. Patient 1. An 86-year-old male with bilateral neovascular AMD returned for scheduled followup. Best corrected visual acuity (BCVA) was 20/100 OD and 20/150 OS. SDOCT revealed cystoid macular edema (CME) OD and solid pigment epithelial detachment (PED) with subretinal fluid OS. The patient was treated with an intravitreal injection of bevacizumab $1.25 \mathrm{mg}$ (Avastin, Genentech, South San Francisco, CA) OD. Nine days following the injection, the patient returned urgently, complaining of metamorphopsia OD. Visual acuity (VA) was 20/60 OD and 20/150 OS, with decreased central macular thickness in each eye (OU) (Figure 1).

2.2. Patient 2. An 86-year-old female with bilateral neovascular AMD returned for scheduled followup. VA with pinhole was 20/70 OD and 20/50 OS. SD-OCT revealed CME and serous PED OD as well as CME OS. The patient was treated with an intravitreal injection of aflibercept $2 \mathrm{mg}$ (Eylea, Regeneron, Tarrytown, NY) OD. Five weeks later, VA with pinhole was 20/40 OU with decreased central macular thickness OU (Figure 2).

2.3. Patient 3. A 74-year-old male with bilateral neovascular AMD returned for scheduled followup. VA with pinhole was 20/25 OD and 20/40 OS. SD-OCT revealed PED OU. The patient was treated with an intravitreal injection of ranibizumab $0.5 \mathrm{mg}$ (Lucentis, Genentech, South San Francisco, CA) OS. At scheduled followup six weeks later, BCVA was 20/20 OD and 20/50 OS with decreased central macular thickness OU (Figure 3). 


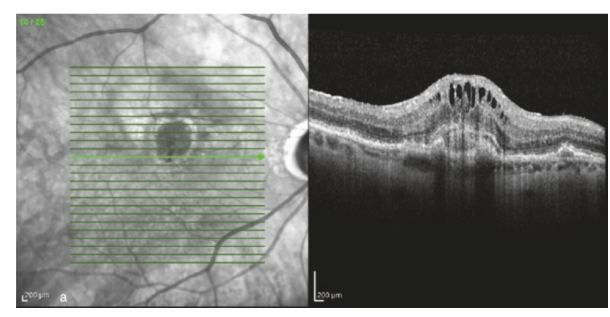

(a)

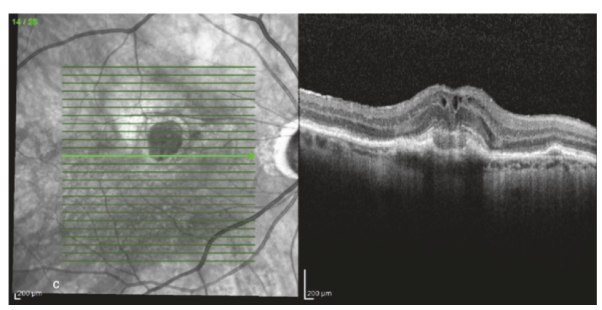

(c)

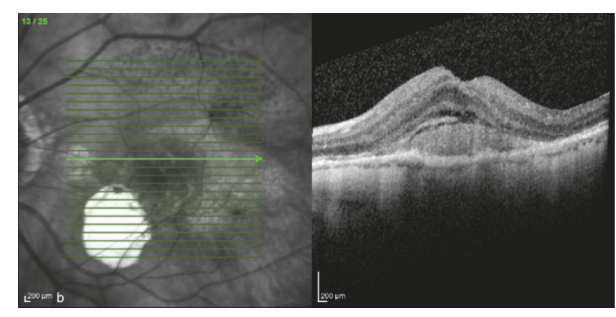

(b)

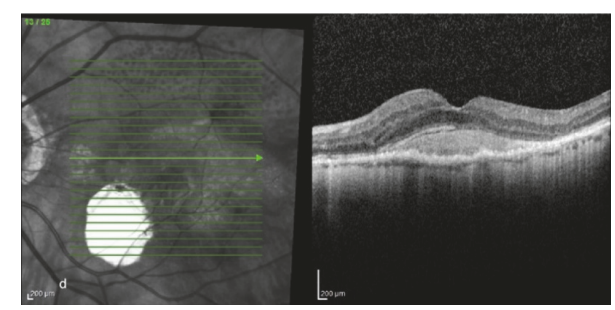

(d)

FIGURE 1: An 86-year-old male with bilateral neovascular age-related macular degeneration (AMD) ((a) and (b)). Nine days following bevacizumab injection OD, there was a reduction in intraretinal and subretinal fluid $\mathrm{OU}((\mathrm{c})$ and $(\mathrm{d}))$.

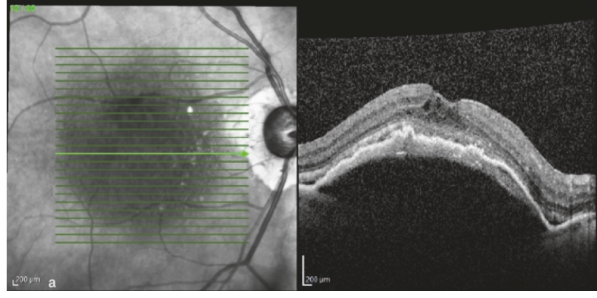

(a)

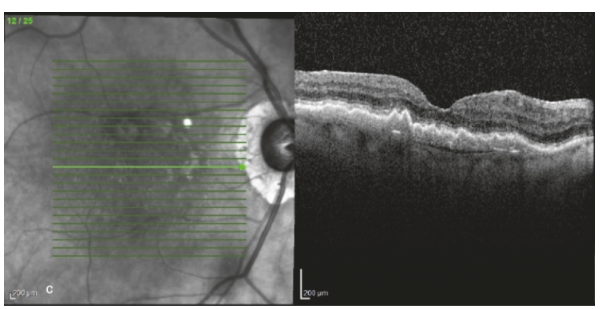

(c)

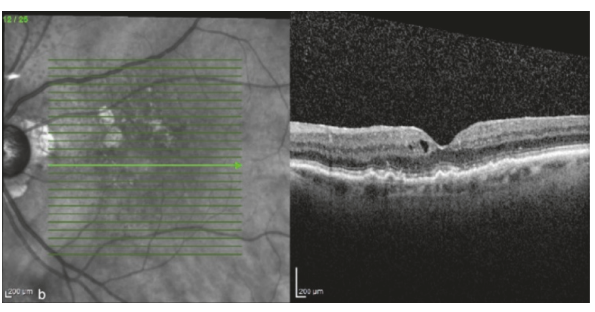

(b)

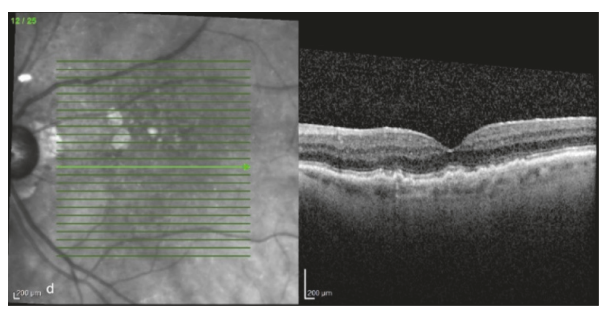

(d)

FIGURE 2: An 86-year-old female with neovascular AMD OU ((a) and (b)). Five weeks following aflibercept injection OD, there was a reduction in intraretinal and subretinal fluid $\mathrm{OU}((\mathrm{c})$ and $(\mathrm{d}))$.

\section{Discussion}

In most patients, unilateral injection of anti-VEGF does not cause a clinically significant effect in the fellow eye. For example, in two large prospective randomized clinical trials with neovascular AMD in one eye and nonneovascular AMD in the fellow eye (Minimally Classic/Occult Trial of the Anti-VEGF Antibody Ranibizumab in the Treatment of Neovascular Age-Related Macular Degeneration [MARINA] and Anti-VEGF Antibody for the Treatment of Predominantly Classic Choroidal Neovascularization in Age-Related Macular Degeneration [ANCHOR]), a retrospective analysis reported no significant differences in the rates of new choroidal neovascularization $(\mathrm{CNV})$ in the nonneovascular fellow eyes of patients randomized to monthly ranibizumab at 12 and 24 months, compared with those randomized to sham injections (MARINA) or photodynamic therapy (ANCHOR) [12]. Similarly, a small prospective series of 26 patients with bilateral neovascular AMD treated with unilateral ranibizumab actually reported a significant increase in central retinal thickness (measured by OCT) in the fellow eye two weeks after injection [13].

However, a bilateral effect following unilateral anti-VEGF injection has been reported previously in some patients with neovascular AMD [3-5], DME [6-10], and uveitic cystoid macular edema [14]. For example, in a retrospective observational series of 25 patients with active neovascular AMD in one eye and disciform scarring in the fellow eye, treatment 


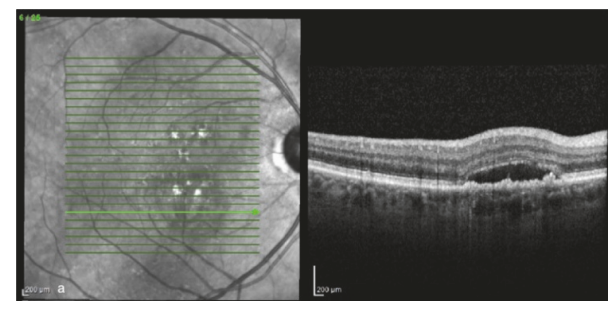

(a)

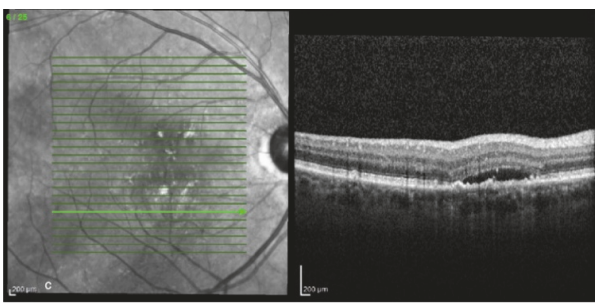

(c)

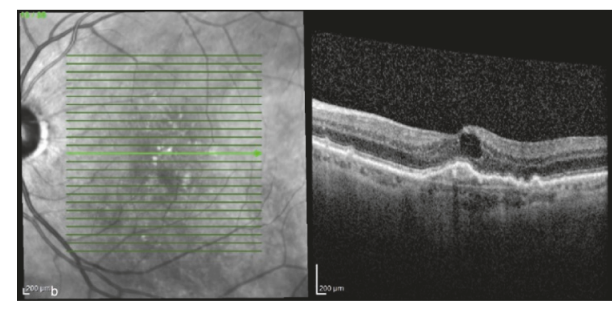

(b)

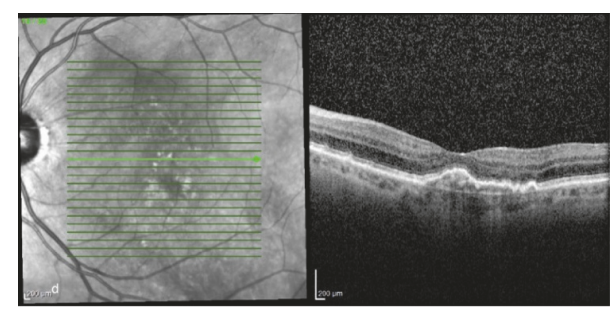

(d)

FIGURE 3: A 74-year-old male with bilateral neovascular AMD ((a) and (b)). Six weeks following intravitreal injection of ranibizumab OS, there was a reduction in intraretinal and subretinal fluid OU ((c) and (d)).

of the eye with active disease with ranibizumab resulted in clinical improvement in the fellow eye with disciform scarring, as measured by visual acuity, fluorescein angiography, and/or OCT [5]. Interestingly, one prospective study of 29 consecutive neovascular AMD patients treated with ranibizumab monthly for three doses reported a significant reduction in the central retinal thickness of the uninjected (nonneovascular) fellow eye [3].

The reason for the therapeutic effect in the untreated fellow eye is unknown but perhaps may be due to the presence of anti-VEGF agents in the systemic circulation [15]. In a prospective study, Avery et al. reported systemic exposure to ranibizumab, aflibercept, and bevacizumab along with associated reduced free VEGF levels, following intravitreal administration in 56 consecutive patients with neovascular AMD [16]. Significantly reduced levels of systemic VEGF were also reported after intravitreal injection of aflibercept and bevacizumab in 14 infants with retinopathy of prematurity [17]. The disruption of the blood-retina barrier in diabetes may enhance this effect [18].

\section{Conclusion}

Here, three such cases are reported in patients with neovascular AMD. These are nonconsecutive, nonrandomized patients who were noted to have this effect. The incidence of this effect is unknown but most likely rare. As further cases are reported, our understanding of this phenomenon should increase.

\section{Conflicts of Interest}

The authors do not have any proprietary conflicts of interest in the materials described in the article. Stephen G. Schwartz, MD, MBA, reports personal fees from Alimera and Welch Allyn. All other authors report no conflicts of interest.

\section{Acknowledgments}

This paper is partially supported by NIH Center Core Grant (no. P30EY014801) and an unrestricted grant from Research to Prevent Blindness (New York, NY).

\section{References}

[1] V. M. Villegas, L. A. Aranguren, J. L. Kovach, S. G. Schwartz, and H. W. Flynn, "Current advances in the treatment of neovascular age-related macular degeneration," Expert Opinion on Drug Delivery, vol. 14, no. 2, pp. 273-282, 2017.

[2] M. W. Stewart, H. W. Flynn, S. G. Schwartz, and I. U. Scott, "Extended duration strategies for the pharmacologic treatment of diabetic retinopathy: current status and future prospects," Expert Opinion on Drug Delivery, vol. 13, no. 9, pp. 1277-1287, 2016.

[3] K. Michalska-Małecka, A. Kabiesz, M. W. Kimsa et al., "Effects of intravitreal ranibizumab on the untreated eye and systemic gene expression profile in age-related macular degeneration," Clinical Interventions in Aging, vol. 11, pp. 357-365, 2016.

[4] Z. Wu and S. R. Sadda, "Effects on the contralateral eye after intravitreal bevacizumab and ranibizumab injections: a case report," Annals, Academy of Medicine, Singapore, vol. 37, no. 7, pp. 591-593, 2008.

[5] A. Rouvas, V. S. Liarakos, P. Theodossiadis et al., "The effect of intravitreal ranibizumab on the fellow untreated eye with subfoveal scarring due to exudative age-related macular degeneration," Ophthalmologica, vol. 223, no. 6, pp. 383-389, 2009.

[6] C. M. Calvo, J. Sridhar, A. Shahlaee, and A. C. Ho, "Reduction of diabetic macular edema in the untreated fellow eye following intravitreal injection of aflibercept," Ophthalmic Surgery, Lasers and Imaging Retina, vol. 47, no. 5, pp. 474-476, 2016.

[7] B. Bakbak, B. Ozturk, S. Gonul, and S. Gedik, "The effect of intravitreal bevacizumab and ranibizumab on macular edema of the contralateral eye: a comparative study of two anti-VEGFs," Oman Journal of Ophthalmology, vol. 9, no. 1, pp. 44-48, 2016. 
[8] K. Matsuyama, N. Ogata, M. Matsuoka, M. Wada, T. Nishimura, and K. Takahashi, "Effects of intravitreally injected bevacizumab on vascular endothelial growth factor in fellow eyes," Journal of Ocular Pharmacology and Therapeutics, vol. 27, no. 4, pp. 379-383, 2011.

[9] J. Hanhart, L. Tiosano, E. Averbukh, E. Banin, I. Hemo, and I. Chowers, "Fellow eye effect of unilateral intravitreal bevacizumab injection in eyes with diabetic macular edema," Eye (Basingstoke), vol. 28, no. 6, pp. 646-653, 2014.

[10] T. Rotsos, C. Symeonidis, I. Triantafillopoulou, S. Kanellopoulos, and A. Kouris, "Significant reduction of diabetic macular edema following intravitreal ranibizumab injection in the fellow eye," International Ophthalmology, vol. 34, no. 6, pp. 1271-1274, 2014.

[11] L. Zlotcavitch, H. W. Flynn, R. L. Avery, and A. Rachitskaya, "Progression to macula-off tractional retinal detachment after a contralateral intraoperative intravitreal bevacizumab injection for proliferative diabetic retinopathy," Clinical Ophthalmology, vol. 9, pp. 69-71, 2015.

[12] I. A. Barbazetto, N. Saroj, H. Shapiro, P. Wong, A. C. Ho, and K. B. Freund, "Incidence of new choroidal neovascularization in fellow eyes of patients treated in the MARINA and ANCHOR trials," American Journal of Ophthalmology, vol. 149, no. 6, pp. 939-el, 2010.

[13] M.-A. Gamulescu and H. Helbig, "Lack of therapeutic effect of ranibizumab in fellow eyes after intravitreal administration," Journal of Ocular Pharmacology and Therapeutics, vol. 26, no. 2, pp. 213-216, 2010.

[14] H. Al-Dhibi and A. O. Khan, "Bilateral response following unilateral intravitreal bevacizumab injection in a child with uveitic cystoid macular edema," Journal of American Association for Pediatric Ophthalmology and Strabismus, vol. 13, no. 4, pp. 400-402, 2009.

[15] F. Semeraro, F. Morescalchi, S. Duse, E. Gambicorti, A. Cancarini, and C. Costagliola, "Pharmacokinetic and pharmacodynamic properties of anti-VEGF drugs after intravitreal injection," Current Drug Metabolism, vol. 16, no. 7, pp. 572-584, 2015.

[16] R. L. Avery, A. A. Castellarin, N. C. Steinle et al., "Systemic pharmacokinetics following intravitreal injections of ranibizumab, bevacizumab or aflibercept in patients with neovascular AMD," British Journal of Ophthalmology, vol. 98, no. 12, pp. 1636-1641, 2014.

[17] C. Huang, R. Lien, N. Wang et al., "Changes in systemic vascular endothelial growth factor levels after intravitreal injection of aflibercept in infants with retinopathy of prematurity," Graefe's Archive for Clinical and Experimental Ophthalmology, vol. 256, no. 3, pp. 479-487, 2018.

[18] C. Zhang, H. Wang, J. Nie, and F. Wang, "Protective factors in diabetic retinopathy: focus on blood-retinal barrier," Discovery Medicine, vol. 18, no. 98, pp. 105-112, 2014. 


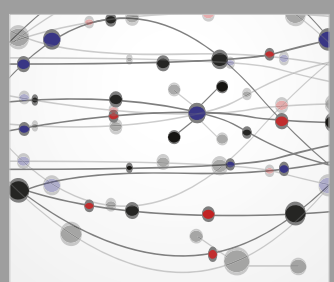

The Scientific World Journal
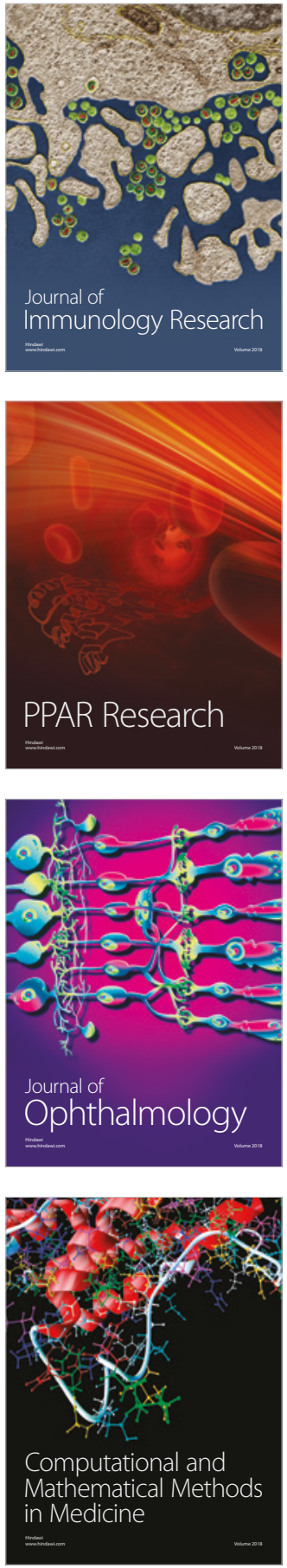

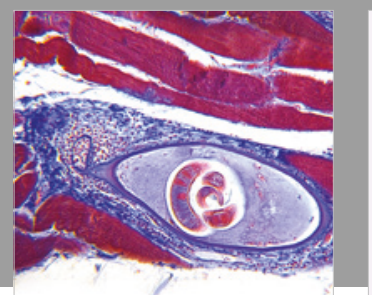

Gastroenterology Research and Practice

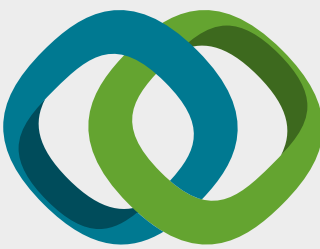

\section{Hindawi}

Submit your manuscripts at

www.hindawi.com
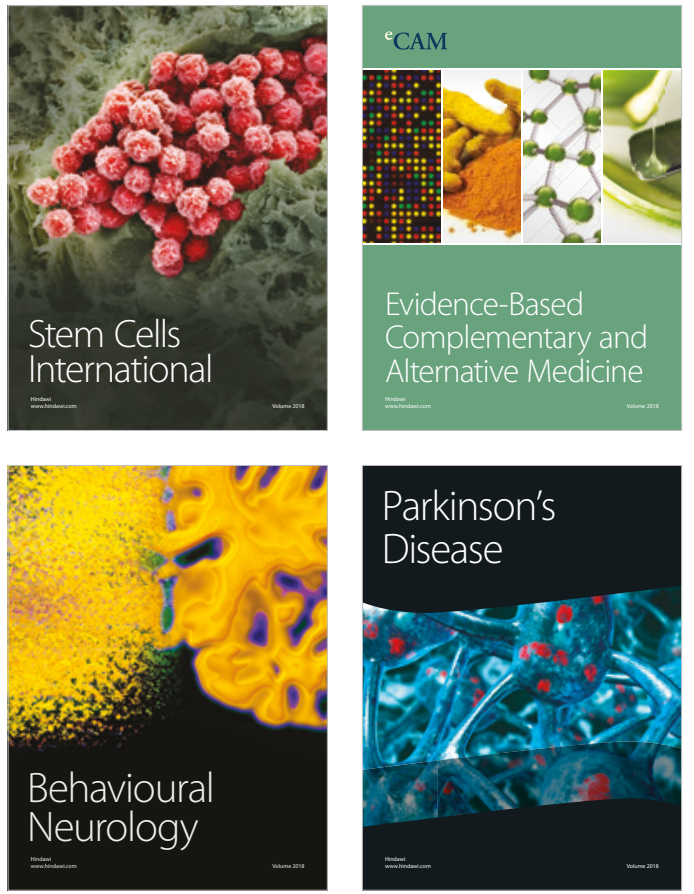

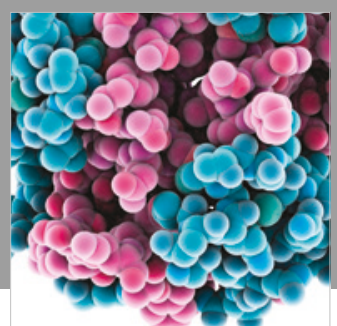

ournal of

Diabetes Research

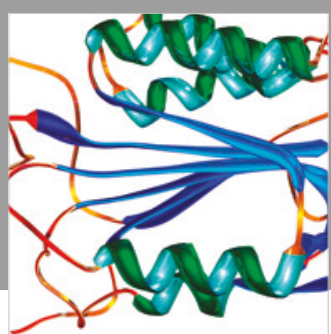

Disease Markers
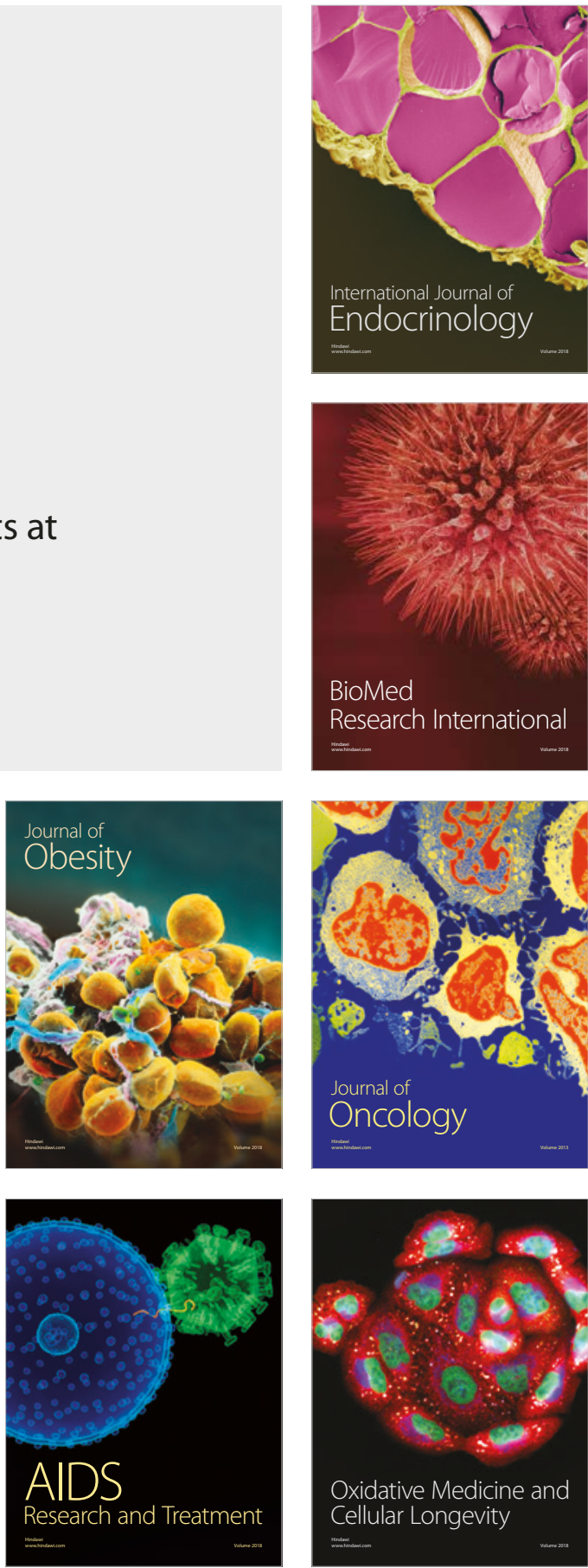